\title{
Genetic Modifications of Voltage-sensitive Sodium Channels in Drosophila: Gene Dosage Studies of the seizure Locus ${ }^{1}$
}

\author{
F. ROB JACKSON, ${ }^{2}$ JANE GITSCHIER, ${ }^{3}$ GARY R. STRICHARTZ, ${ }^{4}$ AND LINDA M. HALL ${ }^{5}$
}

Department of Genetics, Albert Einstein College of Medicine, Bronx, New York 10461

\begin{abstract}
We have identified a genetic locus in Drosophila melanogaster whose product appears to have a structural role in the formation of functional voltage-sensitive sodium channels. This locus, designated seizure, is defined by two temperature-sensitive alleles (sei $i^{t s-1}$ and $s e i^{t s-2}$ ), each of which causes convulsive seizures followed by a rapid but reversible paralysis of adults at restrictive temperatures above $38^{\circ} \mathrm{C}$. Previous work had shown that se $i^{\text {ts-2 }}$ extracts display an altered $\mathrm{pH}$ dependence and an abnormally high $K_{d}$ for $\left[{ }^{3} \mathrm{H}\right]-$ saxitoxin binding at high temperatures, suggesting that sodium channels in se $i^{\text {ts-2 }}$ mutants have an altered structure (Jackson F. R., S. D. Wilson, G. R. Strichartz, and L. M. Hall (1984) Nature 308: 189-191). These binding studies have now been extended to extracts of $s e i^{i s-1}$ which have a $K_{d}$ not significantly different from wild-type at all assay temperatures. However, se $^{i^{\text {ts-1}}}$ extracts show a reduced number of saxitoxin binding sites $\left(B_{\max }\right.$ ) relative to wild-type. This reduction is only 5 to $18 \%$ at $0^{\circ} \mathrm{C}$ but is 17 to $37 \%$ at $39^{\circ} \mathrm{C}$, suggesting that under certain conditions sodium channels in the seits-1 $^{\text {ts }}$ mutant are more labile than those of wild-type. Cytogenetic studies demonstrate that the seizure locus maps within region 60A to 60B8-10 on the second chromosome.
\end{abstract}

Received June 14, 1984; Revised October 11, 1984

Accepted October 24, 1984

${ }^{1}$ We thank Susan Wilson and R. Scott Hawley for helpful discussions, John Fak and John Yee for technical assistance, and Barry Ganetzky for copies of the seits mutants with data regarding their map positions. We also thank Loring Craymer, Georgianna Zimm, and Sandy Rokop for invaluable assistance in locating certain chromosomal rearrangements. A portion of these studies was done in the Department of Biology at Massachusetts Institute of Technology, where J. G. was supported by National Institutes of Health Predoctoral Training Grant GM07287. This work was supported by The Muscular Dystrophy Association and National Institutes of Health Grant NS 16204 to L. M. H. and by National Institutes of Health Grants NS 12828 and 18467 to G. R. S. During a portion of these studies, L. M. H. was a McKnight Scholar in Neuroscience and she is currently an Irma T. HirschlMonique Weill Caulier Career Scientist Awardee. F. R. J. was supported by National Research Service Award NS 07183 from National Institute of Neurological and Communicative Disorders and Stroke.

${ }^{2}$ Present address: Worcester Foundation for Experimental Biology, 222 Maple Avenue, Shrewsbury, MA 01545

${ }^{3}$ Present address: Genentech, Inc., 460 Point San Bruno Boulevard, South San Francisco, CA 94080.

${ }^{4}$ Present address: Anesthesia Research Laboratories, Harvard Medical School, Boston, MA 02115.

${ }^{5}$ To whom correspondence should be addressed, at Department of Genetics, Albert Einstein College of Medicine, 1300 Morris Park Avenue, Bronx, NY 10461.
Gene dosage analysis of $\sim 99.7 \%$ of the genome, including this second chromosome region, failed to detect a wild-type locus whose dose affected saxitoxin-binding activity. Nevertheless, the mutant se $i^{t^{s-2}}$ allele has codominant and dosedependent effects on paralytic behavior and saxitoxin-binding activity. In addition, genetic interactions between $\mathrm{se}^{\mathrm{ts}-2}$ and nap $^{\text {ts }}$ (a nonallelic mutation that reduces number of sodium channels) suggest that seits mutants have enhanced neuronal membrane excitability at the restrictive temperature. These genetic studies, in concert with the biochemical evidence for a structural alteration of sodium channels in se $^{{ }^{\text {ts }}}$ extracts, suggest that the seizure locus encodes a component of the voltage-sensitive sodium channel.

The electrical properties of excitable cells are determined principally by protein channels that selectively pass ions across the cell membrane. Many of these channcls, such as the voltage-sensitive sodium channel, are of interest because they change their permeability properties in response to changes in membrane potential (Hagiwara, 1983; Catterall, 1984). With respect to the sodium channel, biochemical purification coupled with functional reconstitution provide one approach to defining the molecular components that comprise the functional sodium channel complex (Barchi, 1983; Hartshorne and Catterall, 1981, 1984; Miller et al., 1983; Rosenberg et al., 1984a, b; Tamkun et al., 1984; Weigele and Barchi, 1982). While this is a powerful approach, it will not identify factors that are necessary for the processing of channels (Waechter et al., 1983; Bar-Sagi and Prives, 1983), nor will it identify factors involved in the developmental maturation of sodium channels (Baumgold et al., 1983a, b; Strichartz et al., 1983).

A genetic analysis of sodium channels provides a complementary approach which will eventually help to address some of these issues. A first step in this direction was the identification of mutant cell lines with altered sensitivities to sodium channel-specific neurotoxins (West and Catterall, 1979; Costa and Catterall, 1982). These studies identified two classes of sodium channel mutants: those with altered channel structure and those lacking functional sodium channels. Studies on temperature-sensitive paralytic mutants in Drosophila melanogaster have also identified two classes of sodium channel mutants (Jackson et al., 1984). Classical genetic approaches using these paralytic mutants in Drosophila will allow the determination of the number and chromosomal distribution of genes required for the production and developmental regulation of sodium channels.

The genetic properties of mutations affecting ion channels can provide insight into the nature of the wild-type gene product. For example, gene dosage studies of the Shaker locus in Drosophila have provided evidence that certain mutant alleles encode an altered structural component of a potassium channel (Tanuoye et al., 1981). This approach allows the distinction between mutations which reduce channel efficacy by altering channel structure and those which reduce numbers of an otherwise normal channel. 
These studies of potassium channel mutants in Drosophila have utilized an electrophysiological approach to identify genetic modifications of channel function. Our studies have used $\left[{ }^{3} \mathrm{H}\right]$ saxitoxin, which binds selectively to the voltage-sensitive sodium channel (Narahashi, 1972; Ritchie, 1979), as a probe for genetic alterations of sodium channels in temperature-sensitive paralytic mutants (Gitschier et al., 1980; Hall et al., 1982; Jackson et al., 1984). Membrane extracts from one such paralytic mutant, called seizure ${ }^{\text {ts-2 }}\left(\mathrm{se}^{\mathrm{ts}^{\text {s-2 }}}\right)$, exhibited temperature-dependent reductions in $\left[{ }^{3} \mathrm{H}\right]$ saxitoxin-binding activity as a consequence of a reduced affinity of saxitoxin-binding sites (Jackson et al., 1984). Mutant extracts also displayed an abnormal $\mathrm{pH}$ dependence for saxitoxin-binding activity (Jackson et al., 1984). These effects suggested that sodium channels in $\mathrm{se}^{i^{\text {ts-2 }}}$ extracts had an altered structure. We now report gene dosage studies of two different mutant alleles of the seizure locus (seits-1 and $s e i^{i s-2}$. These studies reveal parallel effects of the se $i^{t s-2}$ allele on behavior and saxitoxin-binding activity, and suggest a structural role for the $\mathrm{sei}^{+}$product in sodium channel function. They also suggest that the behavioral phenotype of seits mutants is a consequence of altered sodium channels.

\section{Materials and Methods}

Stocks and culture conditions. Drosophila cultures were grown at $21^{\circ} \mathrm{C}$, except where noted, and on a standard cornmeal medium (Lewis, 1960). Control wild-type Drosophila were the Canton-S strain provided by J. C. Hall (Brandeis University). X-Y translocations were obtained from J. Merriam (University of California, Los Angeles). Y-autosome translocations were provided by L. Craymer (California Institute of Technology), R. Hodgetts (University of Alberta), or D. Lindsley (University of California, San Diego). Second chromosomal deficiencies came from L. Craymer and B. Judd (Research Triangle Park, NC), or the Bowling Green State University Stock Center. All chromosomal rearrangements and mutations except those affecting behavior are described by Lindsley and Grell (1968). The temperature-sensitive para-

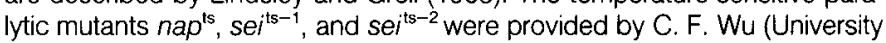
of lowa) and B. Ganetzky (University of Wisconsin). All were ethyl methanesulfonate-induced mutations; nap ${ }^{\text {ts }}$ maps to 2.56 .2 (Wu et al., 1978), whereas the seits mutations both map to 2-106 (B. Ganetzky, personal communication). Although $s c i^{\text {ts }-2}$ is codominant (sce "Results"), scits-1 is clcarly an allele since the paralytic phenotypes of $s e i^{t^{s-1}} / s^{t s-1}, s e^{t s-2} / s e i^{t s-2}$, and heteroallelic se $i^{t s-1} / \mathrm{se}^{\mathrm{ts}-2}$ files are similar, and easily distinguished from $+/ \mathrm{se}^{\mathrm{ts}-2}$ or the wild lype.

Double mutants carrying nap $p^{\text {ts }}$ and $s e i^{\text {ts-2 }}$ were constructed by selecting for recombination between nap $p^{\text {ts }} \mathrm{cn}$ and bw seits-2 chromosomes. To generate $\mathrm{se}^{\mathrm{is}^{\mathrm{s}-2} /+}$ heterozygotes for saxitoxin-binding assays, homozygous mutants were mated to the wild-type Canton-S strain.

Mutants carrying different combinations of $\mathrm{se}^{\mathrm{its}-2}$ and $\mathrm{se}^{+}$were produced using $D p(2 ; Y) b w^{+}$and $\ln (2 L R) 0 . D p(2 ; Y) b w^{+}$(also known as $b w^{+} \eta$ is an insertional translocation of a small region of the second chromosome (salivary region $58 \mathrm{~F} 1-60 \mathrm{~F} 1$ ) into the long arm of the $Y$ chromosome. It contains the se $i^{+}$locus (see "Results"). In(2LR)O is a multiply inverted second chromosome that prevents recombination. It carries $\mathrm{bw}^{+}, \mathrm{se}^{+}$, and the dominant wing mutation Curly $(C y)$. We constructed a stock by mating $X / b W^{+} Y ; b w s e i^{i s-2} /$ $\ln (2 L R) 0$ males to $X / X ; b w$ se $i^{i s-2} / \ln (2 L R) 0$ females. This mating produced the following progeny: $b w^{+} Y$; bw sei $i^{\text {ts-2 }} / b w$ seits-2 (which were seit $/ \mathrm{sei}^{\text {ts-2 }} /$

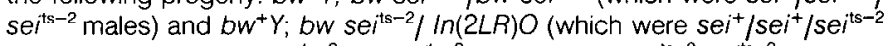
males) as well as bw seits-2 $/ b w$ seits-2 (which were sei $i^{\text {ts }-2} / \mathrm{sei}^{\text {ts }-2}$ females) and bw se $i^{\text {ts-2 }} / / n(2 L R) 0$ (which were se $i^{+} / \mathrm{se}^{\text {ts }-2}$ females). These genotypes could be distinguished on the basis of eye color and wing phenotypes. $A$ comparable stock was constructed for the $\mathrm{se}^{\mathrm{ts}-1}$ allele. Flies carrying either two or three doses of $\mathrm{se}^{+}$were selected from a $\mathrm{cn} b w / \mathrm{cn}$ bw stock in which the males carried three doses and were $b w^{+} \gamma ; c n$ bw/cn bw while the females carried two doses and were $\mathrm{cn}$ bw/cn bw.

Construction of segmental aneuploids. Flies çarrying duplications for segments of autosomes 2 and 3 and their euploid siblings were generated by a technique described by Lindsley et al. (1972). Briefly, aneuploids were constructed by matings between two stocks, each heterozygous for a translocation between the autosome and a genetically marked $Y$ chromosome. The autosomal breakpoints of the two stocks were displaced so that some of the progeny carried either a duplication or a deletion of the material between the breakpoints. Segmental duplications for regions on the $X$ chromosome were constructed using techniques described by Stewart and Merriam (1974). In general, matings were set up so that flies bearing duplications were females; the deletion-bearing males usually did not survive because the deleted segments were large. Flies carrying one or three copies of the small chromosome 4 were generated from matings between wild-type females and males bearing attached- 4 chromosomes.

Table $I$ is a list of the chromosomal rearrangements used to define the cytogenetic position of the seizure locus. A deficiency for salivary chromosome region 59F-60A was synthesized using the reciprocal $Y-2$ translocations $T(Y ; 2) H 143$ and $T(Y ; 2) L 137$ (Lindsley et al., 1972). The proximal element of $T(Y ; 2) H 143$ in combination with the distal element of $T(Y ; 2) L 137$ creates the desired deficiency. The distal elements of $T(Y ; 2) H 143$ and $T(Y ; 2) A 160$ produced duplications that spanned the regions between the $T(Y ; 2)$ breakpoints and the terminus of chromosome $2 \mathrm{R}$.

Preparations of membrane extracts. Membrane extracts were made from homogenates of Drosophila heads as described previously (Gitschier et al., 1980). The upper, lighter colored portion of a pellet from high-speed centrifugation $(40,000 \times g$ for $40 \mathrm{~min})$ of supernatants was resuspended in assay buffer (Gitschier et al., 1980) to a concentration equivalent to $200 \mathrm{mg}$ heads/ $\mathrm{ml}$. Preparations were routinely frozen at $-70^{\circ} \mathrm{C}$ until use. The protein content of extracts was determined according to a modification of the Lowry procedure (Markwell et al., 1978) with bovine serum albumin as the standard.

Toxins. Saxitoxin was tritium-labeled, purified according to the method of Ritchie et al. (1976), and stored at $-76^{\circ} \mathrm{C}$ in $1 \mathrm{~mm} 2$-( $N$-morpholino)ethanesulfonic acid buffer, $\mathrm{pH} 6.5$. The specific activity of $\left[{ }^{3} \mathrm{H}\right]$ saxitoxin used in the majority of experiments was $11.2 \mathrm{dpm} / \mathrm{fmol}$. This batch of toxin gave $K_{d}$ s which were consistently lower and $B_{\max }$ s which were consistently higher than those which we have reported previously using other batches of toxin (Gitschier et al., 1980; Jackson et al., 1984). In all cases reported here, comparisons between different Drosophila stocks were done on the same day using the same batch of $\left[{ }^{3} \mathrm{H}\right]$ saxitoxin. Tetrodotoxin was obtained from Calbiochem or Sigma and stored at $-20^{\circ} \mathrm{C}$ in 20 or $50 \mathrm{~mm}$ citrate buffer, $\mathrm{pH}$ 4.8 .

$\left[{ }^{3} H\right]$ Saxitoxin binding. Saxitoxin-binding assays were performed as described previously (Jackson et al., 1984). The net binding of saxitoxin was calculated by subtracting nonspecifically bound saxitoxin (in the presence of $10 \mu \mathrm{M}$ tetrodotoxin) from the total amount of bound saxitoxin.

To assess the effect of segmental duplications on binding activity, 325 heads from duplication-bearing flies or euploid siblings (aged 3 to $6 \mathrm{~d}$ at $25^{\circ} \mathrm{C}$ ) were homogenized in $0.65 \mathrm{ml}$ of assay buffer by 40 strokes of a Teflon pestle in a glass homogenizer at $1700 \mathrm{rpm}$. Aliquots equivalent to 50 heads were incubated with $5 \mathrm{nM}\left[{ }^{3} \mathrm{H}\right]$ saxitoxin for $1 \mathrm{~h}$ at $4^{\circ} \mathrm{C}$, then filtered as described previously (Jackson et al., 1984). For each genotype, triplicate total and triplicate background measurements were made. To calculate the relative saxitoxin-binding activity, the average amount of saxitoxin bound specifically to extracts equivalent to 50 heads of aneuploid flies was divided by that bound to extracts equivalent to 50 heads of their euploid siblings.

Temperature-induced paralysis. Flies to be tested for temperature sensitivity were gently placed in $25-\mathrm{cm}^{2}$ Corning tissue culture flasks $(\sim 10$ flies/ flask). Locomotor paralysis was observed at the specified temperature by immersing the flasks in a temperature-regulated water bath. The number of flies that had become paralyzed was determined at 15 -sec intervals. Flies were considered to be paralyzed when they were no longer standing. To determine recovery times after paralysis, the flasks were transferred from high temperature to $21^{\circ} \mathrm{C}$.

\section{Results}

The se $i^{\text {ts-2 }}$ has dose-dependent effects on behavior and saxitoxin binding. Gene dosage effects represent one criterion for determining whether a locus encodes a sodium channel component. To look for dosage effects of the seizure ${ }^{t s-2}$ allele, we constructed flies carrying

TABLE I

Summary of deficiencies, duplications, and $Y ; 2$ translocations used to analyze the sei locus

\begin{tabular}{lll}
\hline Aberration & \multicolumn{2}{c}{ Autosomal Breakpoints } \\
\hline$D f(2 R) b w^{5}$ & $59 D 10-\mathrm{E} 1 ;$ & $59 \mathrm{E} 4-\mathrm{F} 1$ \\
$D f(2 R) P x$ & $60 \mathrm{~B} 8-10 ;$ & $60 \mathrm{D} 1-2$ \\
$D f(2 R) P x^{2}$ & $60 \mathrm{C} 5-6 ;$ & $60 \mathrm{D} 9-10$ \\
$D f(2 R) P x^{4}$ & $60 \mathrm{~B}-\mathrm{C} ;$ & $60 \mathrm{D} 1-2$ \\
$D f(2 R) M-c^{33 a}$ & $60 \mathrm{E} 2-3 ;$ & $60 \mathrm{E} 11-12$ \\
$D p(2 ; Y) \mathrm{bw}^{+}$ & $58 \mathrm{~F} 1-59 \mathrm{~A} 2 ;$ & $60 \mathrm{E}-\mathrm{F} 1$ \\
$T(Y ; 2) H 143$ & & $59 \mathrm{~F}$ \\
$T(Y ; 2) L 137$ & & $60 \mathrm{~A}$ \\
$T(Y ; 2) A 160$ & & $60 \mathrm{~B}-\mathrm{C}$ \\
\hline
\end{tabular}



- $s \mathrm{i}^{\mathrm{ts}-2} / \mathrm{sei}^{1 \mathrm{~s}-2}$
$+/$ sei $^{t s-2}$

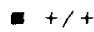
$0+/$ sei $^{t s-2} /$ seits $^{t s} \Delta+/+/$ seits -2

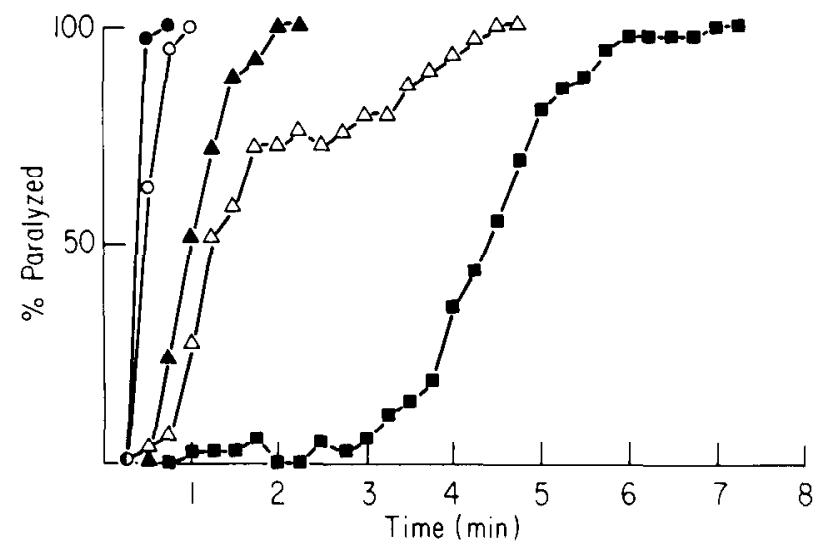

Figure 1. Paralysis of mutants carrying different combinations of $\mathrm{sei}^{+}$ and $s i^{i t s-2}$ alleles. The temperature was $40^{\circ} \mathrm{C}$. Time points are averages of three experiments representing a total of at least 30 flies. Solid symbols are euploid flies; open symbols are aneuploids. - se $^{\text {ts-2 }} / \mathrm{se}^{\mathrm{is}-2}$ females; $\mathrm{O},+1$ $s e i^{t s-2} / s e i^{t s-2}$ males; $\Delta_{,}+/ s^{i t s-2}$ females; $\Delta_{1}+/+/ s e i^{t s-2}$ males; $\boldsymbol{D}_{1}+1+$ males and females.

different ratios of mutant (se $\left.{ }^{i s-2}\right)$ to wild-type $(+)$ alleles, and examined them for paralysis and altered $\left[{ }^{3} \mathrm{H}\right]$ saxitoxin binding at high temperature. Both of these activities are affected in $s e i^{\text {ts-2 }}$ homozygotes. (Jackson et al., 1984). Figure 1 shows that the se $i^{t^{\text {s-2}}}$ mutation has codominant effects on paralytic behavior (A codominant effect is indicated when the phenotype of the heterozygote is intermediate to the phenotypes of the homozygous mutant and homozygous wild-type). At $40^{\circ} \mathrm{C}$, heterozygotes became paralyzed within 2 min at a rate intermediate to that of mutant homozygotes and the wild type. We also studied the behavior of flies which had 3 doses of the seizure locus by using the $Y$-borne duplication $D p(2 ; Y) b w^{+}$that includes $\mathrm{se}^{+}$(B. Ganetzky, personal communication). Males with two copies of $s e i^{t s-2}$ and one of $s e i^{+}\left(+/ s e i^{t s-2} / s e i^{\text {ts-2 }}\right)$ became paralyzed more slowly than sibling females with only two copies of $\mathrm{se}^{\mathrm{ts}-2}\left(\mathrm{se}^{\mathrm{ts}-2} / \mathrm{se}^{\mathrm{ts}-2}\right)$ (Fig. 1). This behavioral difference was enhanced further when the temperature was one degree lower; at $39^{\circ} \mathrm{C}$, the time for paralysis of $50 \%$ of the population was $19.7 \pm$ 0.71 (SE) sec for $\mathrm{se}^{\mathrm{ts}-2} / \mathrm{se}^{\mathrm{ts}-2}$ males compared to $40.7 \pm 1.3 \mathrm{sec}$ for $+/ \mathrm{se}^{i^{t s}-2} / \mathrm{se}^{\mathrm{ts}-2}$ males. When all of the genolypes shown in Figure 1 are considered, it is obvious that $s e i^{\text {ts }-2}$ affects behavior in a dose-dependent manner. As the dose of the mutant allele was increased relative to the wild-type allele, flies became paralyzed in increasingly shorter periods of time. These effects on behavior are not a consequence of changing the number of copies of the $\mathrm{sei}^{+}$ locus. In control experiments, flies carrying two $(+/+)$ or three $(+/$ $+/+$ ) doses of $\mathrm{sei}^{+}$displayed identical paralytic behavior. If the se $i^{\text {ts }-2}$ mutation simply reduced or abolished a gene product, then flies of the genotype $+/+/ \mathrm{se}^{\mathrm{ts}-2}$ would be wild-type, since they carry the normal number (two) of $\mathrm{se}^{+}$alleles. The observation that $+/+/$ se $^{\text {ts }-2}$ flies became paralyzed faster than the wild-type (Fig. 1) suggests that the $\mathrm{se}^{\mathrm{is}-2}$ allele specifies an altered gene product rather than simply reducing the amount of product.

The se $i^{\text {ts-2 }}$ allele affects behavior in a codominant and dosedependent manner (Fig. 1). If the allele is also responsible for the abnormal saxitoxin-binding seen in mutant extracts, then it should have dose-dependent effects on binding activity. It is known from previous work (Jackson et al., 1984), that the se $i^{\text {ts-2 }}$ mutation decreases the affinity of channels for saxitoxin (i.e., increases $K_{d}$ ) at $40^{\circ} \mathrm{C}$, but does not affect the number of binding sites. If flies with a mixture of wild-type and mutant genes produce both normal and mutant channels, then when binding is measured at a concentration equivalent to the $K_{d}$, an apparent reduction in the amount of saxitoxin bound, which is proportional to the relative amount of mutant channels, will be seen. Figure 2 shows that this is the case. At a concentration of saxitoxin equivalent to the $K_{d}$ for wild-type saxitoxin binding, $+/ \mathrm{se}^{\mathrm{ts}-2},+/ \mathrm{se}^{\mathrm{ts}-2} / \mathrm{se}^{\mathrm{ts}-2}$, and $\mathrm{se} i^{\mathrm{ts}-2} / \mathrm{se}^{i \mathrm{ts}-2}$ extracts had $85.8,69.0$, and $57.2 \%$ of the wild-type activity, respectively. Thus, the reduction in binding activity is directly correlated with the relative dose of the mutant se $i^{1 s-2}$ allele. These dose dependent effects, when considered with the evidence for a structural alteration of sodium channels in homozygous se ${ }^{i s-2}$ mutants, suggest that the se $i^{\text {ts-2 }}$ allele specifies an abnormal sodium channel.

The sei locus maps within region 60A-60B8-10 of chromosome 2. Because of the dosage effects seen for the se $i^{\text {ts-2 }}$ allele, we were interested in obtaining a more accurate cytogenetic map position for the sei locus. Results from the use of duplication $D p(2 ; \gamma) b^{+}$ placed the sei locus within region 58F1-60F1 of the second chromosome. We have further localized the $s e i^{\text {ts-2 }}$ mutation within this region using the duplications $(D p)$ and deficiencies $(D f)$ described in Table I. Flies carrying $s e i^{t^{s-2}}$ in combination with each of the deficiencies shown diagrammatically in Figure 3 were tested for paralysis at $38-39^{\circ} \mathrm{C}$. None of the sei $i^{\text {ts-2 }} / D f$ combinations resulted in paralysis within 2 min. Therefore, all of these deficiency-bearing chromosomes retain the sei+ locus.

We also examined homozygous seits-2 mutants carrying each of the duplications shown in Figure 3. As mentioned previously, $D p(2 ; Y) b w^{+}$contains a copy of $\mathrm{sei}^{+}$. The terminal duplication $D p(2 R) D, H 143$, according to a similar behavioral criterion, also carries $\mathrm{sei}^{+}$. Therefore, the sei locus must reside in $59 \mathrm{~F}$ or to the right. Because $D f(2 R) 59 F-60 A$, which has the same left breakpoint as $D p(2 R) D, H 143$, does not uncover sei, the locus must be within $60 \mathrm{~A}$ or to the right. $D p(2 R) D, A 160$ lacks sei $i^{+}$since $s e i^{\mathrm{ts}-2} / \mathrm{se}^{\mathrm{ts}-2} /$ $D p(2 R) D, A 160$ mutants become paralyzed in a manner identical to their $s e i^{t s-2} / s e i^{t s-2}$ siblings (data not shown). Hence, sei is within $60 \mathrm{C}$ or to the left. However, Df(2R)Px deletes $60 \mathrm{C}$ and does not uncover the sei locus. Therefore, the locus is to the left of 60B8-10.

Analysis of another allele of the seizure locus. Analysis of multiple alleles at a genetic locus can often provide insights regarding the nature of that locus. Another mutation $\left(s e i^{\text {ts-1 }}\right.$ ) that caused temperature-dependent paralysis in adults had been defined as an allele of $\mathrm{se}^{\mathrm{is}-2}$ (C. F. Wu and B. Ganetzky, personal communication), and this has been confirmed by us. We characterized seits-1 behaviorally and biochemically to compare its properties to $s i^{\text {ts }-2}$. In contrast to $s e i^{t s-2}$, the sei $i^{i s-1}$ allele is recessive with respect to paralysis. Thus, $+/ \mathrm{se}^{\mathrm{ts}^{\mathrm{s}-1}}$ heterozygotes became paralyzed in a manner indistinguishable from wild-type (Fig. 4). Even when flies carried two doses of seits-1 and only onc dosc of the sei $i^{+}$allele, their paralytic behavior was indistinguishable from the wild type. In comparison, flies carrying

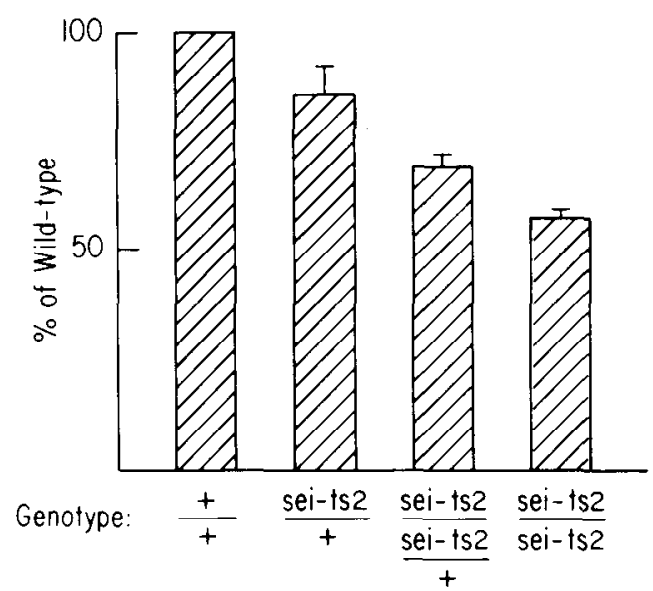

Figure 2. Dosage effects of $\mathrm{sei}^{\mathrm{ts}-2}$ on $\left[{ }^{3} \mathrm{H}\right]$ saxitoxin-binding activity. Saxitoxin-binding activity was examined at a concentration of toxin equivalent to the wild-type $K_{d}$. Assays were performed at $40^{\circ} \mathrm{C}$. Saxitoxin bound in extracts from the genotypes shown was expressed as a percentage of wildtype. Histograms are means \pm the range of two experiments. 


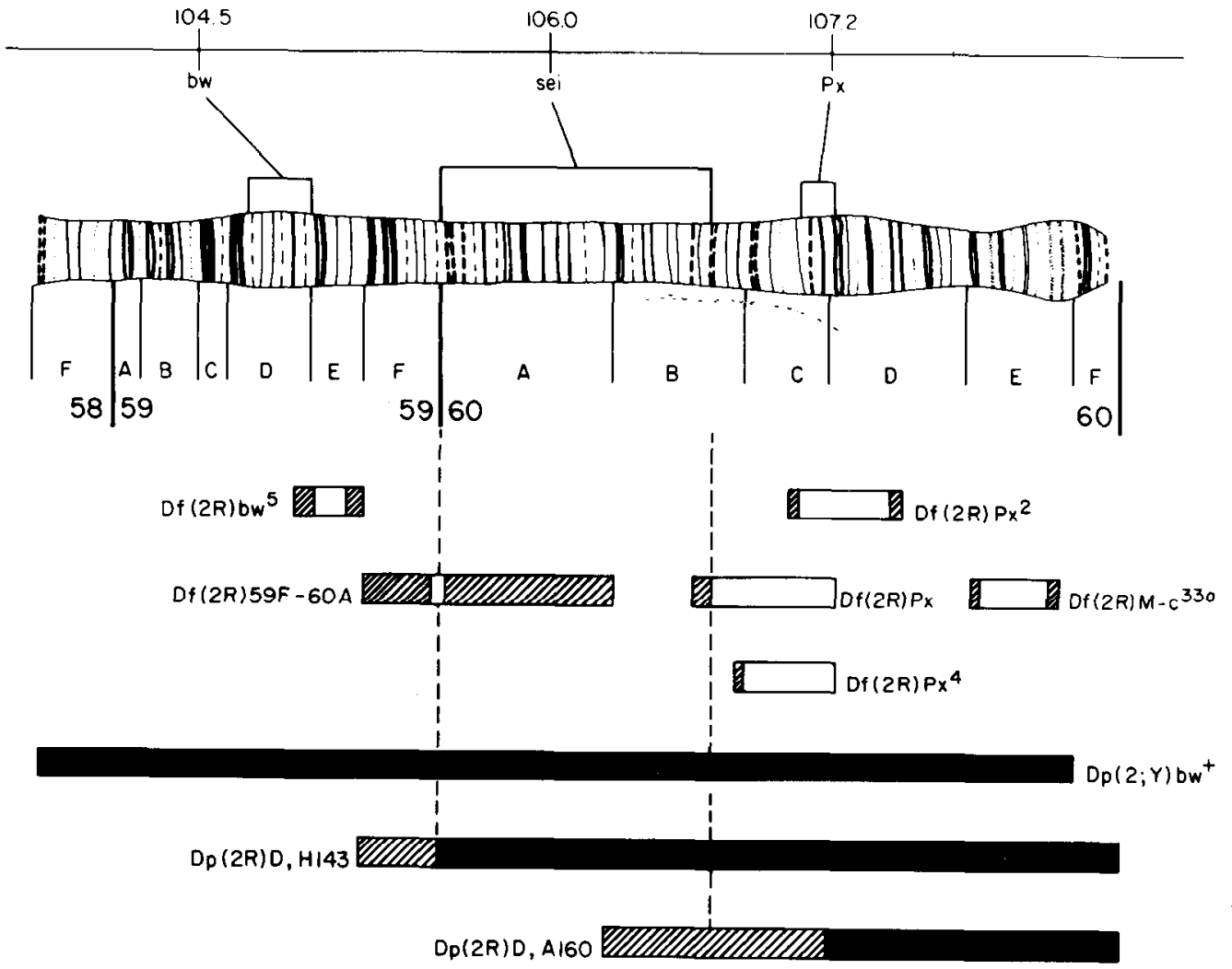

Figure 3. Diagrammatic representation of the tip of the right arm of chromosome 2 summarizing genetic and cytogenetic analyses of the sei locus. The positions of sei and two other loci (bw and $P x)$, as determined from recombination and cytogenetic mapping, are indicated at the top. The chromosomal locations of deficiencies $(D f)$ and duplications $(D p)$ are shown by open and solid bars, respectively. The maximal lengths of duplications are shown. Ambiguity with respect to locations of breakpoints is indicated by cross-hatching. $D f(2 R) 59 F-60 A$ is a synthetic deficiency composed of the proximal element of translocation $T(Y ; 2) H 143$ and the distal element of $T(Y ; 2) L 137$. $D p(2 R) D, H 143$ and $D p(2 R) D, A 160$ are terminal duplications produced from the distal elements of $T(Y ; 2) H 143$ and $T(Y$; ) A 16O, respectively. $D f(2 R) 59 F-$ $60 A$ and $D P(2 R) D, H 143$ have the same proximal breakpoint. Chromosomal banding patterns for this portion of chromosome 2 were redrawn from Bridges's revised reference map as illustrated in Lindsley and Grell (1968). Some of the bands drawn as single lines are actually doublets according to Bridges.

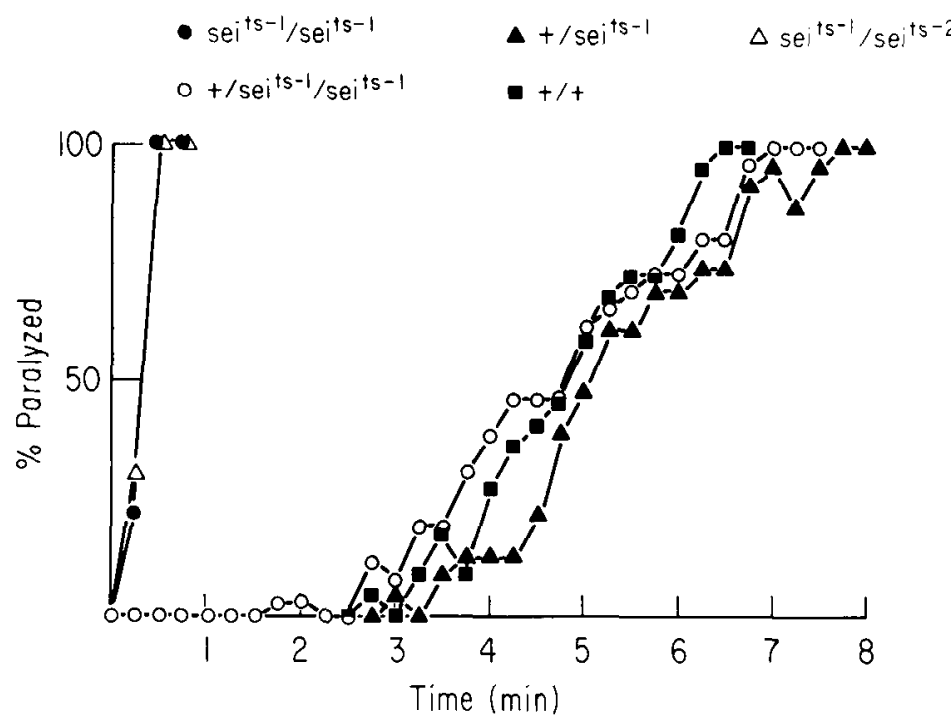

Figure 4. Temperature-induced paralysis of $s i^{t^{s-1}}$ mutants versus the wild-type. The temperature was $40.5^{\circ} \mathrm{C}$, and each time point represents at least 26 flies. - sei $^{i s-1}$ homozygotes; $O,+/ \mathrm{se}^{t s-1} /$ sei ${ }^{i s-1} ; \boldsymbol{\Delta},+/$ sei $^{i s-1}$ heterozygotes; $\Delta$, sei $^{i s-1} / \mathrm{sei}^{t s-2} ; \mathbf{\square},+1+$ wild type. Stocks were grown at $25^{\circ} \mathrm{C}$.

the heteroallelic combination $\left(s e i^{\text {ts }-1} / s^{i t s-2}\right)$ were indistinguishable from either seits-1 or seits-2 homozygotes at $40^{\circ} \mathrm{C}$.

The $\left[{ }^{3} \mathrm{H}\right]$ saxitoxin binding properties of $\mathrm{se}^{\mathrm{is}-1}$ extracts also differ from those of $s e i^{t s-2}$. Extracts of $s e^{i t s-2}$ had an abnormally high $K_{d}$ for saxitoxin binding at $39^{\circ} \mathrm{C}$ when compared to wild-type, but a normal $K_{d}$ at $0^{\circ} \mathrm{C}$. The seits-2 mutant extracts also displayed an aitered $\mathrm{pH}$ dependence for toxin-binding (Jackson et al., 1984). This altered $\mathrm{pH}$ dependence was not seen in se ${ }^{\mathrm{ts}-1}$ extracts (data not shown). However, when saturation binding curves were done at $39^{\circ} \mathrm{C}$ and at $\mathrm{pH} 7.0$, several different extracts of se $i^{\text {ts-1 }}$ showed reductions in the number of saturable binding sites $\left(B_{\max }\right)$ ranging from 17 to $37 \%$. Results from a typical experiment are illustrated in the Scatchard plot (Scatchard, 1949) shown in Figure 5. This seits-1 extract had about $24 \%$ fewer saxitoxin-binding sites than the control wild-type extracl when both were assayed at $39^{\circ} \mathrm{C}$. The average $B_{\max }$ in four sets of extracts was $218.4 \pm 8.4(\mathrm{SE}) \mathrm{fmol} / \mathrm{mg}$ protein

and $165.3 \pm 8.4 \mathrm{fmol} / \mathrm{mg}$ protein for the wild-type and $\mathrm{se}^{\mathrm{ts}-1}$, respectively. This difference in $B_{\max }$ is statistically significant $(t=4.4$; $p<0.005)$. At $39^{\circ} \mathrm{C}$, the sei $i^{\text {ts-1 }}$ allele shows a small but statistically insignificant effect on the $K_{d}$ for saxitoxin binding (wild-type $=1.66$ $\pm 0.13 \mathrm{~nm}$ and $\mathrm{se} i^{\mathrm{ts}-1}=1.36 \pm 0.14 \mathrm{~nm}$ ). In all experiments at $0^{\circ} \mathrm{C}$, the $K_{\alpha} s$ for $s e i^{t s-1}$ and the wild type were similar. In the $0^{\circ} \mathrm{C}$ experiment shown in Figure 5 , the se $i^{\text {is-1 }}$ extract had only $5 \%$ fewer saxitoxin binding sites than the wild type. In another $0^{\circ} \mathrm{C}$ experiment, however, a different seits-1 extract had $18 \%$ fewer binding sites than wild-type.

No single wild-type locus has dosage effects on saxitoxin binding. Since it appears that the sei locus encodes a component of the sodium channel, we were interested in determining whether an increase in the number of wild-type se $i^{+}$genes caused a corresponding increase in the number of saxitoxin-binding sites. Using the duplication $D p(2 ; Y) b w^{+}$, we compared toxin binding activities in 


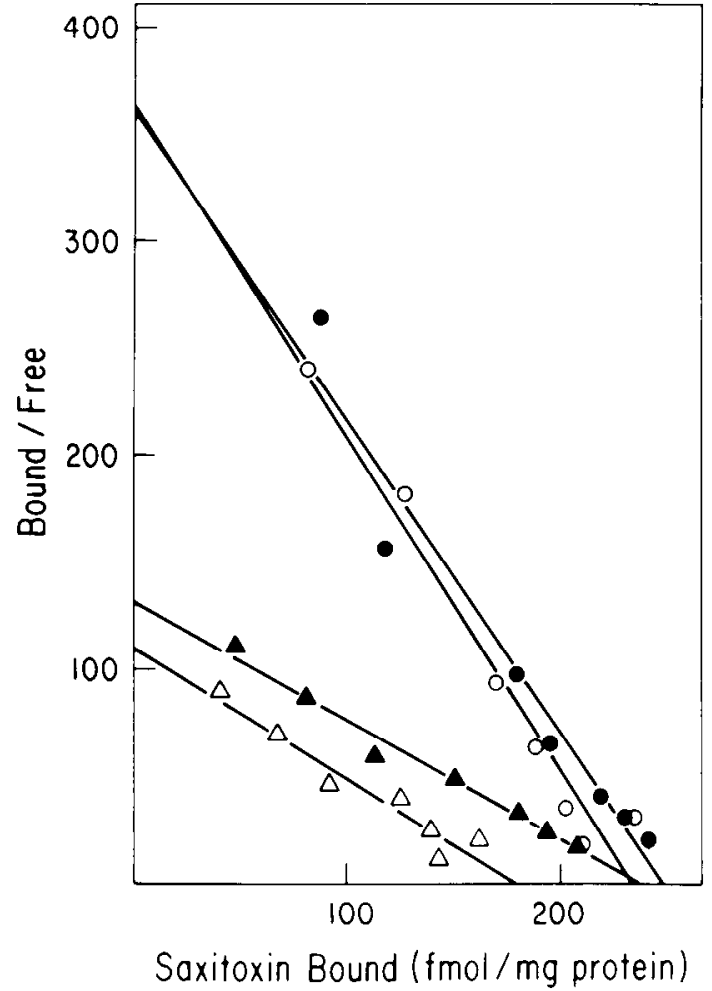

Figure 5. Scatchard analysis of $\left[{ }^{3} \mathrm{H}\right]$ saxitoxin-binding activity in seist and wild-type extracts. Assays were performed at $0^{\circ} \mathrm{C}$ and $39^{\circ} \mathrm{C}$ as described previously (Jackson et al., 1984), and data were plotted after the manner of Scatchard (1949). X-intercepts indicate the number of saturable saxitoxinbinding sites $\left(B_{\max }\right)$ in preparations. The slope of lines is the negative reciprocal of the $K_{\sigma}$ for saxitoxin binding. The binding parameters are as follows: wild-type at $0^{\circ} \mathrm{C}$ where $K_{d}=0.68 \mathrm{nM}$ and $B_{\max }=248.1 \mathrm{fmol} / \mathrm{mg}$ protein; $O$, sei $i^{i s-1}$ at $0^{\circ} \mathrm{C}$ where $K_{o}=0.65 \mathrm{nM}$ and $B_{\max }=234.8 \mathrm{fmol} / \mathrm{mg}$ protein; $\boldsymbol{\Lambda}$, wild-type at $39^{\circ} \mathrm{C}$ where $K_{d}=1.82 \mathrm{nM}$ and $B_{\max }=238.4 \mathrm{fmol} / \mathrm{mg}$ protein; and $\triangle$, seits-1 at $39^{\circ} \mathrm{C}$ where $K_{\sigma}=1.67 \mathrm{nM}$ and $B_{\max }=181.9 \mathrm{fmol}$ mg protein.

males carrying three doses of $\mathrm{sei}^{+}$with their euploid $\left(\mathrm{Sei}^{+} / \mathrm{Sei}^{+}\right)$ female siblings and with Canton-S wild-type males (also seit $/ \mathrm{sei}^{+}$). There were no significant differences between the genotypes. Assuming that the sei locus encodes a sodium channel component, these results could be considered surprising, since a direct correlation between gene number and product activity has been demonstrated for many proteins in Drosophila (see Greenspan, 1980, and references therein). Therefore, we constructed segmental duplications (Lindsley et al., 1972) to determine if there was a wild-type locus that had dosage effects on saxitoxin binding activity. An examination of virtually the entire genome ( $99.7 \%)$ failed to identify such a locus. Figure 6 illustrates a comparison of saxitoxin-binding activities in duplication-bearing flies carrying three doses $(+/+/+)$ of a particular region relative to their euploid siblings carrying two doses $(+/+)$ of the same region. If a region exerts a dosage effect on binding activity, the theoretical expectation is that duplication-bearing flies will have $3 / 2=1.5$ times the activity of their euploid sibs. None of the duplications resulted in the expected $50 \%$ elevation in binding activity. For all regions, the variations were within the error of measurement. Possible reasons for the lack of dosage effect for a wild-type locus are considered in detail in the "Discussion."

Behavioral evidence of enhanced membrane excitability displayed by seits mutants. Although we have not characterized seits mutants electrophysiologically, behavioral data suggest that the paralytic behavior is not simply due to an inactivation of channels at high temperatures. When exposed to high temperature, seits mutants become hyperactive and exhibit convulsive seizures before becoming paralyzed. In addition, their subsequent rate of recovery at permissive temperatures is strongly dependent on the length of prior paralysis; the longer the time of paralysis, the slower the rate of recovery (Fig. 7 ). The seits-2 mutation also affects this behavior in a codominant manner; the recovery of $\mathrm{se}^{\mathrm{is}-2} /+$ heterozygotes takes only half as long as the recovery of $\mathrm{se}^{\mathrm{ts}-2} / \mathrm{se}^{\mathrm{ts}-2}$ homo not shown). These phenotypes might be the result of an abnormal activation of sodium channels during exposure to high temperature. If so, these phenotypes should be suppressed by factors which reduce the number of functional sodium channels.

The nap ${ }^{\text {ts }}$ mutation which reduces the number of saxitoxin-binding sites (Hall et al., 1982; Jackson et al., 1984) and causes a temperature-dependent blockade of action potentials (Wu et al., 1978; Wu and Ganetzky, 1980) provides a genetic way to test this model. By constructing the nap seits-2 double mutant, we can genetically reduce the number of saxitoxin binding sites in $s e i^{t^{t-2}}$ flies. As shown in Figure 7, nap ${ }^{\text {ts }}$ does suppress the recovery phenotype of $s e i^{t s-2}$. Double mutants that are homozygous for both nap ${ }^{\text {ts }}$ and $s e i^{\text {ts }-2}$ display recovery times that are more rapid than those seen in the $\mathrm{se}^{i^{\text {ts }}-2}$ population (Fig. 7). Three stocks of nap ${ }^{\text {ts }} \mathrm{sei}^{\text {ts }-2}$ double mutants, each established from an independently isolated recombinant chromosome, yielded comparable results. Hence, nap ${ }^{\text {ts }}$ accelerates recovery times, presumably by reducing sodium current. These results are consistent with the suggestion that sodium channels in se $i^{\text {is }}$ mutants have enhanced activity at high temperature. The recovery times of the se $i^{\text {ts }-2}$ mutant are accelerated in the presence of nap ${ }^{\text {ts }}$ possibly because nap ${ }^{\text {ts }}$ reduces the number of functional sodium channels, and this modulates the effect of enhanced channel activation at high temperature.

\section{Discussion}

We have presented genetic and biochemical evidence that the sei locus encodes a structural element of the functional sodium channel complex. The se $i^{\text {ts-2 }}$ mutation exerts codominant and dosedependent effects on sodium channels and behavior. In fact, the severity of both phenotypes is correlated with the relative dose of the se $^{i s-2}$ allele. The gene dosage effects of this allele suggest that the mutation is affecting sodium channel properties by altering a stoichiometric rather than a catalytic process. One explanation for these genetic results is that the mutant allele encodes a variant polypeptide that still comprises part of the sodium channel complex. As the number of mutant genes increases relative to the wild type, there would be a proportionate increase in the variant polypeptide relative to the normal one with corresponding effects on channel function.

This type of genetic rationale has been used by others to suggest that mutant alleles affecting other ionic currents encode structural components of channels. For example, certain Shaker mutations, which atfect the $\mathrm{Ca}^{2+}$-independent $\mathrm{K}^{+}$current of Drosophila, are codominant and have dose-dependent effects on $\mathrm{K}^{+}$current (Tanouye et al., 1981). The Shaker locus has been postulated to be a potassium channel structural gene (Tanouye et al., 1981). In addition, although most mutations affecting $\mathrm{Ca}^{2+}$ currents in Paramecium are recessive, mutations called dancers are codominant and have been postulated to specify a variant structural component of the $\mathrm{Ca}^{2+}$ channel complex (Hinrichsen and Saimi, 1984; Hinrichsen et al. 1984).

Although we propose that the $s e i^{+}$locus codes for a structural component of sodium channels, the $s i^{t^{t-1}-1}$ and $s e i^{\text {ts-2 }}$ alleles have dissimilar effects on behavior and saxitoxin-binding activity; seits-1 homozygotes are noticeably more "hyperactive" than seits-2 mutants even at permissive temperatures (unpublished observations). The se $i^{\text {ts-1 }}$ allele has recessive effects on paralytic behavior, whereas $s e i^{t s-2}$ is codominant. Finally, se $i^{t s-1}$ mutant extracts have reduced numbers of saxitoxin-binding sites when assayed at either high or low temperatures; however, the reduction is more dramatic at high temperatures. In contrast, se $i^{\text {ts-2 }}$ extracts retain the wild-type number of binding sites, but saxitoxin-binding sites have a lower affinity at high temperatures and have a $\mathrm{pH}$ sensitivity which is different from that of the wild type (Jackson et al., 1984). These differential effects 


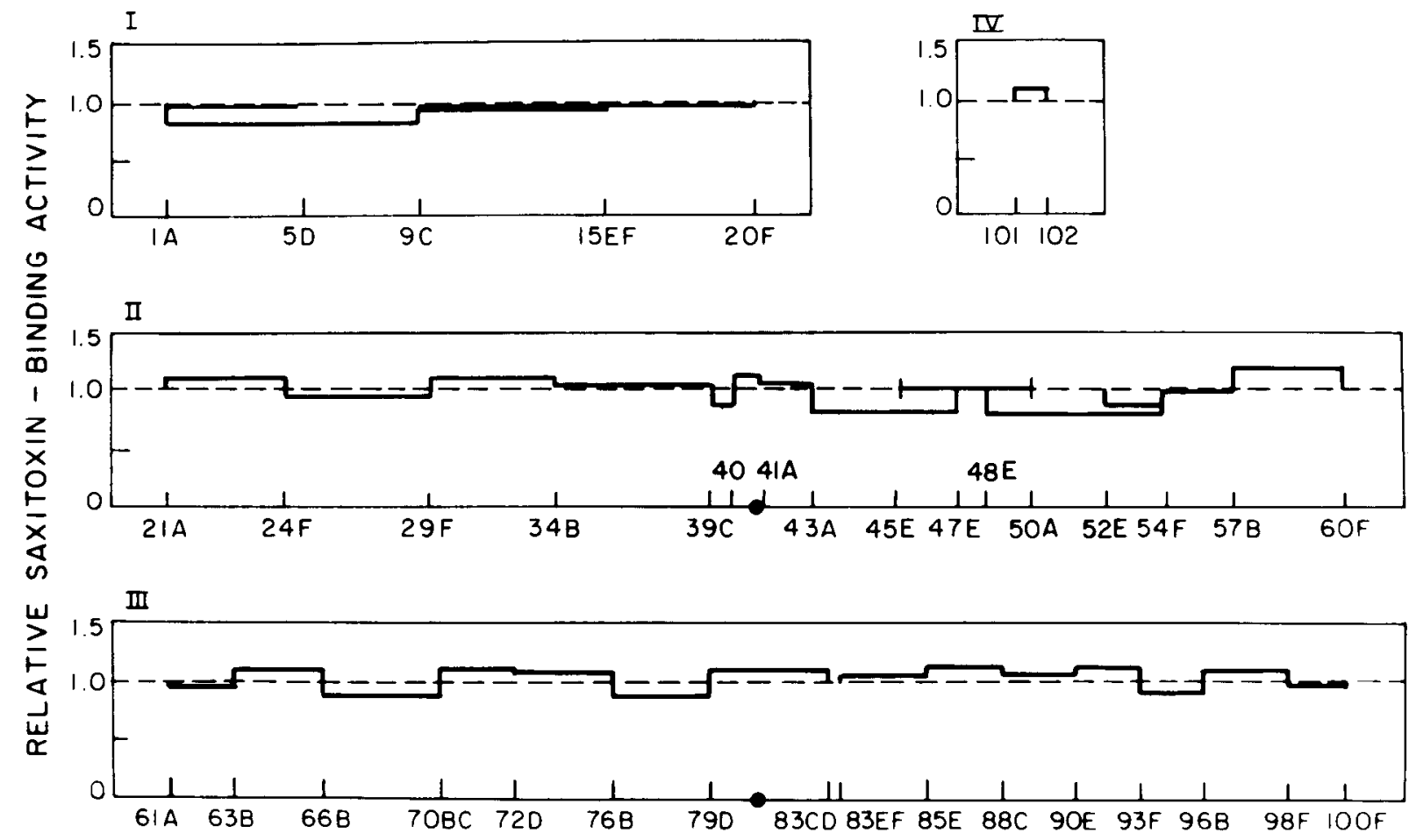

Figure 6. Effects of segmental duplications on the number of saxitoxin-binding sites in Drosophila. Flies bearing segmental duplications and their euploid siblings were generated and assayed for binding activity as described under "Materials and Methods." The amount of specific saxitoxin-binding activity in segmentally duplicated flies was normalized to that in euploids. The relative saxitoxin-binding activity in the duplicated flies is plotted as a function of the location of the duplicated segments on the chromosomes (I, II, III, IV), given in units of cytological bands of polytene chromosomes (Lindsley and Grell, 1968).

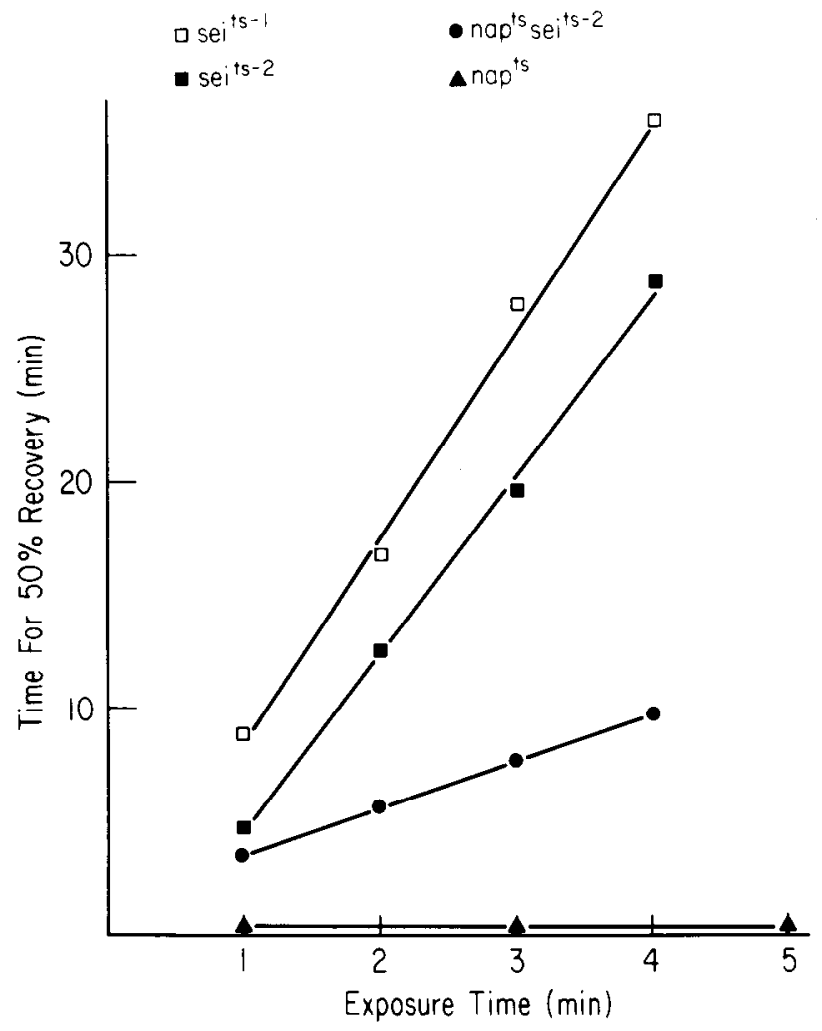

Figure 7. Recovery of temperature-sensitive paralytic mutants at $21^{\circ} \mathrm{C}$ after exposure to $40^{\circ} \mathrm{C}$ for different periods of time. Time points are averages of two to three experiments. Mutants were homozygous for nap ${ }^{t s}(\mathbf{A}), s^{i s-1}$ $(\square), \operatorname{sei}^{t s-2}(\square)$, or both nap $p^{t s}$ and $\operatorname{sei}^{t s-2}(\mathbf{Q})$ on behavior and saxitoxin binding may result because $s e i^{\text {ts-1 }}$ and seits-2 affect different structural domains of the sodium channel.

Any model that attempts to explain the role of the $s e i^{+}$product in sodium channel function must incorporate our observations which suggest that $s e i^{\text {ts }}$ mutants have enhanced membrane excitability. Even at permissive temperatures, seits mutants are abnormally active. When they are shifted from the permissive to the nonpermissive temperature, they exhibit hyperexcitability and convulsions which lead to paralysis. This behavior is similar to symptoms seen in insects poisoned by agents which cause enhanced excitability by binding to sodium channels to produce a modified open state (Hille, 1968; Narahashi and Haas, 1967; Narahashi, 1982). Using patch clamp techniques in Drosophila (Wu et al., 1983), it may be possible to determine directly if single sodium channels in $s e i^{\text {ts }}$ mutants exhibit abnormal openings at high temperatures.

Assuming that the sei locus encodes a component of the sodium channel, it might seem surprising that the wild-type sei $i^{+}$allele does not exert dosage effects on saxitoxin-binding activity. In fact, there is not a single wild-type locus in the entire Drosophila genome that has dosage effects on sodium channel number (Fig. 6). There are several possible explanations for these results. One possibility is that the structural gene for the saxitoxin binding component resides in the third chromosome region $83 \mathrm{CD}-83 \mathrm{EF}$ which is lethal when three doses are present. This region represents less than $0.3 \%$ of the genome (see Fig. 6), and to date no putative sodium channel mutations have been mapped to it. However, with the genetic tools currently available, we cannot exclude this possibility.

Another possibility is that a posttranscriptional regulation of sodium channel mRNA levels modulates the effects expected for an increase in sodium channel structural gene number. Osley and Hereford (1981) have shown that for yeast histone genes an extra copy of the $\mathrm{H} 2 \mathrm{~A}, \mathrm{H} 2 \mathrm{~B}$ gene pair does not increase the steady-state levels of histone mRNA. This steady-state regulation is a consequence of an increased degradation of histone mRNA, and gene dosage effects are seen only if the rate of $\mathrm{H} 2 \mathrm{~A}, \mathrm{H} 2 \mathrm{~B}$ gene transcription is measured. 
Alternatively, extra channel protein may be synthesized in response to an increase in gene dosage, but would not be detected in our assay (which measures only membrane-bound channels) if the number of membrame sites for sodium channel insertion is limiting. In addition to posttranscriptional and insertional regulation, it is possible that other cellular processes accurately regulate the number of sodium channels that become membrane bound. Indeed, work of Sherman and Catterall (1984) suggests that a constant level of membrane excitability is ensured by a $\mathrm{Ca}^{2+}$-mediated regulation of sodium channel number in cultured rat muscle cells.

Finally, the absence of gene dosage effects might result if the Drosophila sodium channel, like that of mammals (Hartshorne and Catterall, 1984), is a multimeric protein composed of several different polypeptides. If the genes encoding the polypeptides map to different regions of the genome, then a duplication for one region would not necessarily result in an increase in total channel number, since the subunit(s) encoded by nonduplicated regions would be limiting. Distinguishing among the above possibilities will require further genetic and molecular analysis of sodium channcls.

Since we postulate that the seizure locus encodes a structural component of the voltage-sensitive sodium channel, it is interesting to consider the experiments required to test definitively that suggestion. The most rigorous test would require the demonstration that seizure mutations alter the primary amino acid sequence in a functional channel component. Alternatively, if only a single protein is required for sodium channel function in Drosophila as it is for Electrophorus (Rosenberg et al., 1984a, b), then cloning the seizure ${ }^{+}$ gene and demonstrating channel function in a transformation assay (Gunderson et al., 1984) would also provide convincing evidence that seizure is the sodium channel structural gene.

Although not definitive, there are, in addition, other predictions that can be made regarding the characteristics the seizure locus should have if it is a sodium channel structural gene. For example, the gene dosage studies predict that a deletion heterozygote for the seizure locus will show reduced saxitoxin binding relative to wildtype. Furthermore, all sodium channels characterized to date contain a large $\left(M_{r}=230,000\right)$ protein. If the seizure locus were the structural gene for such a protein, it might present a large target for chemical mutagenesis and additional mutant alleles should be readily obtainable. In addition, since this large channel protein contains at least four pharmacologically distinct sites which define different functional domains of the molecule, different mutant alleles of the structural gene for this protein should show distinctly different phenotypes depending on which domain of the sodium channel (i.e., the ion pore, the activation gate, etc.) is mutationally altered. Work in progress aimed at isolating new seizure alleles by chemical mutagenesis and by transposon tagging (Bingham et al., 1982) should provide further insight to the molecular nature of the seizure locus and its relationship to the voltage-sensitive sodium channel.

\section{References}

Barchi, R. L. (1983) Protein componerits of the purified sudium channel from rat skeletal muscle sarcolemma. J. Neurochem. 40: 1377-1385.

Bar-Sagi, D., and J. Prives (1983) Tunicamycin inhibits the expression of surface sodium channels in cultured muscle cells. J. Cell Physiol. 114: $77-81$.

Baumgold, J., J. B. Parent, and I. Spector (1983a) Development of sodium channels during differentiation of chick skeletal muscle in culture. I. Binding studies. J. Neurosci. 3: 995-1003.

Baumgold, J., J. B. Parent, and I. Spector (1983b) Development of sodium channels during differentiation of chick skeletal muscle in culture. $11 .{ }^{22} \mathrm{Na}^{+}$ uptake and electrophysiological studies. J. Neurosci. 3: 1004-1013.

Bingham, P. M., M. G. Kidwell, and G. M. Rubin (1982). The molecular basis of P-M hybrid dysgenesis: The role of the $P$ element, a P-strain-specific transposon family. Cell 29: 995-1004.

Catterall, W. A. (1984) The molecular basis of neuronal excitability. Science 223: 653-661.

Costa, M. R., and W. A. Catterall (1982) Characterization of variant neuroblastoma clones with missing or altered sodium channels. Mol. Pharmacol. 22: $196-203$.
Gitschier, J., G. R. Strichartz, and L. M. Hall (1980) Saxitoxin binding to sodium channels in head extracts from wild-type and tetrodotoxin-sensitive strains of Drosophila melanogaster. Biochim. Biophys. Acta 595: 291303.

Greenspan, R. J. (1980) Mutations of choline acetyltransferase and associated neural defects in Drosophila melanogaster. J. Comp. Physiol. 137: 83-92.

Gunderson, C. B., R. Miledi, and I. Parker (1984) Messenger RNA from human brain induces drug. and voltage-operated channels in Xenopus oocytes. Nature 308: 421-424.

Hagiwara, S. (1983) Membrane Potential-Dependent lon Channels in Cell Membrane, Raven Press, New York

Hall, L. M., S. D. Wilson, J. Gitschier, N. Martinez, and G. R. Strichartz (1982) Identification of a Drosophila melanogaster mutant that affects the saxitoxin receptor of the voltage-sensitive sodium channel. Ciba Found. Symp. 88: 207-220.

Hartshorne, R. P., and W. A. Catterall (1981) Purification of the saxitoxin receptor of the sodium channel from rat brain. Proc. Natl. Acad. Sci. U. S. A. 78: $4620-4624$.

Hartshorne, R. P., and W. A. Catterall (1984) The sodium channel from rat brain: Purification and subunit composition. J. Biol. Chem. 259: 16671675.

Hille, B. (1968) Pharmacological modifications of the sodium channels of frog nerve. J. Gen. Physiol. 51: 199-219.

Hinrichsen, R. D., and Y. Saimi (1984) A mutation that alters properties of the calcium channel in Paramecium tetraurelia. J. Physiol. (Lond.) 351: 397-410.

Hinrichsen, R. D., Y. Saimi, and C. Kung (1984) Mutants with altered $\mathrm{Ca}^{2+}$ channel properties in Paramecium tetraurelia: Isolation, characterization and genetic analysis. Genetics 108: 545-558.

Jackson, F. R., S. D. Wilson, G. R. Strichartz, and L. M. Hall (1984) Two types of mutants affecting voltage-sensitive sodium channels in Drosophila melanogaster. Nature 308: 189-191.

Lewis, E. B. (1960) A new standard food medium. Drosophila Information Service 34: 117-118.

Lindsley, D. L., and E. H. Grell (1968) Genetic Variations of Drosophila melanogaster, Carnegie Institution of Washington, Publication No. 627.

Lindsley, D. L., L. Sandler, B. S. Baker, A. T. C. Carpenter, R. E. Dennell, J C. Hall, P. A. Jacobs, G. L. G. Miklos, B. K. Davis, R. C. Gethmann, R. W. Hardy, A. Hessler, S. M. Miller, H. Nozawa, D. M. Parry, and M. Gould Somero (1972) Segmental aneuploidy and the genetic gross structure of the Drosophila genome. Genetics 71: 157-184.

Markwell, M. A. K., S. M. Haas, L. L. Bieber, and N. E. Tolbert (1978) A modification of the Lowry procedure to simplify protein determination in membrane and lipoprotein samples. Anal. Biochem. 87: 206-210.

Miller, J. A., W. S. Agnew, and S. R. Levinson (1983) Principal glycopeptide of the tetrodotoxin/saxitoxin binding protein from Electrophorus electricus: Isolation and partial chemical and physical characterization. Biochemistry 22: $462-470$.

Narahashi, T. (1972) Mechanism of action of tetrodotoxin and saxitoxin on excitable membranes. Fed. Proc. 31: 1124-1132.

Narahashi, T. (1982) Modification of nerve membrane sodium channels by the insecticide pyrethroids. Comp. Biochem. Physiol. [C] 72: 411-414.

Narahashi, T., and H. G. Haas (1967) DDT: Interaction with nerve membrane conductance changes. Science 157: 1438-1440.

Osley, M. A., and L. M. Hereford (1981) Yeast histone genes show dosage compensation. Cell 24: 377-384.

Ritchie, J. M. (1979) A pharmacological approach to the structure of sodium channels in myelinated axons. Annu. Rev. Neurosci. 2: 341-362.

Ritchie, J. M., R. B. Rogart, and G. R. Strichartz (1976) A new method for labelling saxitoxin and its binding to non-myelinated fibres of the rabbit vagus, lobster walking leg, and garfish olfactory nerves. J. Physiol. (Lond.) 261: 477-494.

Rosenberg, R. L., S. A. Tomiko, and W. S. Agnew (1984a) Reconstitution of neurotoxin-modulated ion transport by the voltage-regulated sodium channel isolated from the electroplax of Electrophorus electricus. Proc. Natl. Acad. Sci. U. S. A. 81: 1239-1243.

Rosenberg, R. L., S. A. Tomiko, and W. S. Agnew (1984b) Single channel properties of the reconstituted voltage-regulated $\mathrm{Na}$ channel isolated from the electroplax of Electrophorus electricus. Proc. Natl. Acad. Sci. U. S. A. 81: $5594-5598$.

Scatchard, G. (1949) The attractions of proteins for small molecules and ions. Ann. N. Y. Acad. Sci. 51: 660-672.

Sherman, S. J., and W. A. Catterall (1984) Electrical activity and cytosolic 
calcium regulate levels of tetrodotoxin-sensitive sodium channels in cultured rat muscle cells. Proc. Natl. Acad. Sci. U. S. A. 81: 262-266.

Stewart, B. R., and J. R. Merriam (1974) Segmental aneuploidy and enzyme activity as a method for cytogenetic localization in Drosophila melanogas ter. Genetics 76: 301-309.

Strichartz, G. R., D. Bar-Sagi, and J. Prives (1983) Differential expression of sodium channel activities during development of chick skeletal muscle cells in culture. J. Gen. Physiol. 82: 365-384.

Tamkun, M. M., J. A. Talvenheimo, and W. A. Catterall (1984) The sodium channel from rat brain: Reconstitution of neurotoxin-activated ion flux and scorpion toxin binding from purified components. J. Biol. Chem. 259: 1676-1688.

Tanouye, M. A., A. Ferrus, and S. C. Fujita (1981) Abnormal action potentials associated with the Shaker complex locus of Drosophila. Proc. Natl. Acad. Sci. U. S. A. 78: 6548-6552.

Waechter, C. J., J. W. Schmidt, and W. A. Catterall (1983) Glycosylation is required for maintenance of functional sodium channels in neuroblastoma cells. J. Biol. Chem. 258: 5117-5123.

Weigele, J. B., and R. L. Barchi (1982) Functional reconstitution of the purified sodium channel protein from rat sarcolemma. Proc. Natl. Acad. Sr.i. U. S. A. 79: 3651-3655.

West G. J., and W. A. Catterall (1979) Selection of variant neuroblastoma clones with missing or altered sodium channels. Proc. Natl. Acad. Sci. U. S. A. 76: 4136-4140.

Wu, C. - F., and B. Ganetzky (1980) Genetic alteration of nerve membrane excitability in temperature-sensitive paralytic mutants of Drosophila melanogaster. Nature 286: 814-816.

Wu, C. -F., B. Ganetzky, L. Y. Jan, Y. -N. Jan, and S. Benzer (1978) A Drosophila mutant with a temperature-sensitive block in nerve conduction. Proc. Natl. Acad. Sci. U. S. A. 75: 4047-4051

Wu, C. -F., N. Suzuki, and M. -M. Poo (1983) Dissociated neurons from normal and mutant Drosophila larval central nervous system in cell culture. J. Neurosci. 3: 1888-1899. 